\title{
NewComerCorner
}

Joane Amrhein*

\section{Leichte Sprache und Klart Språk}

\author{
Ein Vergleich eines deutschen und eines norwegischen Konzeptes für leicht verständliche \\ Sprache
}

https://doi.org/10.1515/iwp-2019-2058

Zusammenfassung: Dieser Artikel behandelt die Konzepte „Leichte Sprache“ (LS) im Deutschen und „Klart Språk“ (KS) im Norwegischen. Dabei werden die jeweiligen Zielgruppen identifiziert und die öffentliche Meinung zu den Konzepten aufgezeigt. Die Unterschiede zwischen den Zielgruppen sind deutlich sichtbar. „Leichte Sprache“ wurde zur Inklusion eingeführt und zielt primär auf Menschen mit Lernschwäche ab. „Klart Språk“ soll für mehr Demokratie im Land sorgen und richtet sich daher an die ganze Bevölkerung. Eine Studie zeigte, dass die Zielgruppe „Leichte Sprache“ überwiegend positiv bewertet, auch wenn nicht alle Regeln für sinnvoll erachtet werden. Jenseits davon wird aber kontrovers diskutiert, ob „Leichte Sprache“ die ganze Bevölkerung ansprechen sollte oder nur bestimmte Gruppen. Auch „Klart Språk“ findet vorwiegend große Zustimmung, besonders in Regierungsund Verwaltungskreisen. Dennoch gibt es innerhalb der norwegischen Gesellschaft zwei Personengruppen, für die der Einatz von „Klart Språk“ kontrovers diskutiert wird. Das sind zum einen Menschen mit Norwegisch als Fremdsprache und solche, denen das Lesen schwerfällt.

Deskriptoren: Sprache, Grammatik, Vergleich, Zielgruppe, Leichte Sprache, Einfache Sprache, Klart Språk

\section{Leichte Sprache and Klart Språk}

Comparison of a German concept and a Norwegian concept of plain language

\footnotetext{
Abstract: This article deals with the concept "Leichte Sprache" (LS) in German and "Klart Språk" (KS) in Norwegian. The focus groups of the two concepts and the public opinion about them will be shown. The differences between the two concepts and their focus groups are clear. "Leichte Sprache" was established because of the thought of inclusion and is primarily aimed at mentally disabled

*Kontaktperson: Joane Amrhein, Hochschule Darmstadt, University of Applied Sciences, Max-Planck-Straße 2, 64807 Dieburg,

E-Mail: joane.amrhein@stud.h-da.de
}

people. "Klart Språk" is justified by helping the democracy and therefore aimed at the whole nation. A study shows, that the opinion of the focus group on "Leichte Sprache" is mainly positive, even if they disapprove of some current rules. Nonetheless there are discussions within the society whether or not "Leichte Sprache" should be for everybody. "Klart Språk" is also approved by a lot of people, especially within the government and administration. Nevertheless, within the Norwegian Society there are two groups of people for whom the use of "Klart Språk" is controversially discussed. First are people with Norwegian as second language, to whom "Klart Språk" could be helpful. Second are people with difficulties in reading.

Descriptors: Language, Grammar, Comparison, Target Group, Easy Language, Simple Language, Klart Språk

\section{Leichte Sprache et Klart Språk}

Une comparaison d'un concept allemand et un concept norvégien pour une langage facile à lire et à comprendre

Résumé: L'article s'occupe avec les deux concepts „Leichte Sprache“ (LS) en allemand et „Klart Språk“ (KS) en norvégien. Le groupe-cible de chaque concept et l'opinion publique de la société sont l'objet du texte. Les différences entre les deux concepts et leurs groupes-cible sont évident. „Leichte Sprache“ a été établé à cause de l'inclusion des handicapés mental et vise cette clientèle. „Klart Språk“ doit aider avec la democratie et pour cette raison vise tout le peuple. Une recherche montre que l'opinion du groupe-cible de „Leichte Sprache“ est en majorité positif, pourtant il y a aussi quelques règles qui ne sont pas autant acceptes. Malgré cela les opinions divergent à ce sujet. Ils ne se mettent pas d'accord si le concept doit etre pour tout le monde ou juste pour les handicapés mental. KS est aussi plutôt accepté, surtout chezlegrouvernement etl'administration. Deux groups de personnes qui remettent le concept en question sont personnes avec norvégien comme langue étrangère et personnes qui ne peuvent pas lire correctement.

Descripteurs: Langue, Grammaire, Comparaison, Groupe cible, Langage facile, Langage simple, Klart Språk 


\section{Einleitung und Motivation}

Über 20 Millionen Menschen ${ }^{1}$ in Deutschland zwischen 18 und 64 Jahren fällt das Lesen und Schreiben schwer. Darunter sind 14,5 Prozent, die nicht richtig lesen oder schreiben können, und weitere 25,9 Prozent, die selbst gängige Wörter als schwer zu schreiben empfinden (vgl. Grotlüschen und Riekmann, 2011). Daher ist es sinnvoll, einen Weg zu finden, wie auch mit diesen Menschen effektiv kommuniziert werden kann. Da Inklusion und Gleichberechtigung eine immer größere Rolle spielen, ist es wichtig, auch in der Sprache für Barrierefreiheit zu sorgen.

Das Partnerprojekt „Aspects of Linguistic Complexity“ der Hochschule Darmstadt und der Universität Trondheim befasst sich mit „Leichter Sprache“ im Deutschen und „Klart Språk“ im Norwegischen, um ein regelbasiertes System für die automatische Übersetzung von „Leichter Sprache“ und „Klart Språk“ aufzubauen (vgl. Hochschule Darmstadt, 2018).

Dieser Artikel soll eine Einführung in die beiden Konzepte „Leichte Sprache“ (LS) und „Klart Språk“ (KS) geben und aufzeigen, für wen diese gedacht sind. Dabei werden eventuelle Unterschiede der beiden Zielgruppen herausgearbeitet und gezeigt, wer noch von den Konzepten profitieren kann und wie die Öffentlichkeit diese bewertet. Es wird unterschieden zwischen Menschen mit und ohne Lernschwäche.

\section{Klärung der Begriffe}

\section{Leichte Sprache}

„Leichte Sprache ist eine sehr leicht verständliche Sprache. Man kann sie sprechen und schreiben." (Netzwerk Leichte Sprache, o.A.)

„[...] eine Form barrierefreier Kommunikation [...]“" (Bock, 2018)

Es gibt weder eine Definition, noch ist der Begriff Leichte Sprache (LS) ein geschützter Begriff. Deshalb kommt es häufig vor, dass LS mit Einfacher Sprache (ES) verwechselt oder synonym verwendet wird. Das kann zum einen daran liegen, dass beides Konzepte sind, mit denen die deutsche Sprache vereinfacht werden soll. Zum ande-

1 Zur leichteren Lesbarkeit wurde hier eine neutrale Ausdrucksweise gewählt und nur in Einzelfällen das generische Maskulinum verwendet. Es sollen sich jedoch alle Geschlechter angesprochen fühlen. ren haben sie ähnliche Zielgruppen. ES richtet sich, vergleichbar mit KS, an eine breitere Masse der Gesellschaft. Während LS vorwiegend zur Inklusion gedacht ist. LS basiert meist auf Regeln, für Einfache Sprache gibt es kein festes Regelwerk. Zudem ist die Erscheinungsform verschieden. LS sieht vor, hinter jedem Satz einen Absatz zu machen, um ein besseres Leseverständnis und einen besseren Überblick über den Text zu haben. Auch sollen zusammengesetzte Wörter mit einem Bindestrich kenntlich gemacht werden. Einfache Sprache hat eine freiere Form, die keine Absätze oder ähnliches vorschreibt (vgl. Bundeszentrale für politische Bildung, 2014).

Doch die Regeln für LS werden diskutiert. Bettina Bock ist der Meinung, man solle die bestehenden Regeln als Richtlinien sehen und diese nicht unbedingt einhalten. (Bock, 2018)

Die Idee einer einfach verständlichen Sprache ist nicht nur in Deutschland und Norwegen zu finden. Die Webseite clarity-international.net listet insgesamt 25 Vertreter aus verschiedenen Ländern der Welt, die ähnliche Konzepte haben (vgl. Clarity, o.A.). Allerdings können diese sich, wie LS und KS, voneinander in den Konzepten und der Umsetzung unterscheiden.

\section{Klart Språk}

Anders als für LS gibt es für Klart Språk, auch genannt Klarspråk (KS), eine Definition. Diese wurde von der internationalen Arbeitsgruppe IPLWG (International Plain Language Working Group) herausgearbeitet und besagt: „Klarspråk er kommunikasjon med så tydelig ordlyd, struktur og visuell utforming at leserne i målgruppen finner informasjonen de trenger, forstår den og kan bruke den." (Språkrådet, 2013a) Zu Deutsch: „Klare Sprache ist Kommunikation mit einer so deutlichen Formulierung, Struktur und visuellen Gestaltung, dass die Leser der Zielgruppe die benötigten Informationen finden, verstehen und nutzen können.“ (vgl. Språkrådet, 2013a)

Auf der Webseite des Sprachenrates, Språkrådet, finden sich folgende fünf Eigenschaften, die KS haben soll (vgl. Språkrådet, 2013b):

1. „Klare Sprache fördert Demokratie und Rechtsstaatlichkeit.“ Hiermit ist gemeint, dass sich bei einer klaren und deutlichen Verwaltungssprache der Regierung mehr Menschen an der Demokratie beteiligen können. Sie müssen nicht das Gefühl haben, dass sie durch unverständliche Texte ausgeschlossen werden.

2. „Klare Sprache schafft Vertrauen.“ Wenn der Adressat den Text nicht versteht, kann schnell Misstrauen entstehen. KS schafft Vertrauen in den Sender der Nach- 
Tabelle 1: Richtlinien für Texte in LS (nach Baumert, 2016; Freyhoff, 1998).

\begin{tabular}{lll}
\hline Verwenden & Vermeiden & Vorsicht \\
\hline Einfache, unkomplizierte Sprache & $\begin{array}{l}\text { Abstrakte Begriffe (,Rabeneltern“, } \\
\text { „Zahn der Zeit“, etc.) }\end{array}$ & Leser auf respektvolle Weise ansprechen! \\
\hline Kurze Worte, aus der Alltagsprache & $\begin{array}{l}\text { Querbezüge (Eine Frau spricht mit ihrer } \\
\text { Katze. Sie heißt Nele.) }\end{array}$ & Vorhandenes Wissen nicht überschätzen! \\
\hline Oft persönliche Ansprache & Konjunktiv & Bei Zahlen! \\
\hline Praktische Beispiele & Fremdwörter & \\
\hline Meistens kurze Sätze & $\begin{array}{l}\text { Fachjargon, Abkürzungen und Initialen } \\
\text { (Ausnahmen: WC, PKW) }\end{array}$ & \\
\hline Positive Sprache (keine doppelten & $\begin{array}{l}\text { Ungebräuchliche Redewendungen und } \\
\text { Metaphern („Das ist doch Schnee von } \\
\text { Verneinungen) }\end{array}$ & \\
\hline Ehestern.“) & \\
\hline Immer die gleichen Begriffe & & \\
(nicht: „Auto“, „Vehikel“, „Kiste“, & \\
„Fahrzeug“ in einem Text) & \\
\hline Einfache Zeichensetzung & \\
\hline Nur einen Gedanken pro Satz & \\
\hline Anweisungen für den Text & \\
\hline
\end{tabular}

richt, da dieser mit leichten und verständlichen Texten auf den Adressaten eingeht.

3. „Klare Sprache spart Zeit und Geld.“ Indem die Texte einfacher verständlich sind, gibt es weniger Rückfragen. Das bedeutet weniger Bearbeitungsaufwand für die Regierung.

4. „Klare Sprache fördert Kommunikation.“ Denn nun haben beide Seiten eine Möglichkeit, einfacher miteinander zu kommunizieren.

5. „Die Öffentlichkeit soll Vorbild sein" und mit gut verständlicher Sprache Missverständnisse aus dem Weg räumen. Denn komplizierte Verwaltungssprache wird teilweise so aufgefasst, als ob der Staat etwas zu verbergen hätte. Dies soll mit KS verhindert werden.

\section{Regeln}

\section{Leichte Sprache}

Da es für Leichte Sprache (LS) unterschiedliche Regelansätze gibt, die sich in den meisten Punkten überschneiden, wird in Tabelle 1 ein Ansatz exemplarisch dargestellt. Das Regelwerk des Vereins Netzwerk Leichte Sprache ist etwas detaillierter und hat mehr Vorschriften.

\section{Klart Språk}

Für KS sind die Regeln nicht ganz so strikt. Der Sprachenrat hat hierfür zehn Richtlinien aufgestellt, die man beim Schreiben eines Textes in KS beachten sollte.

1. Werden Sie sich bewusst, warum Sie etwas schreiben wollen und für wen.

2. Schreiben Sie direkt an den Empfänger.

3. Finden Sie heraus, wer schreibt. ${ }^{2}$

4. Verwenden Sie aktive Formulierungen - finden Sie heraus, wer was tut.

5. Fangen Sie mit dem Wichtigsten an.

6. Machen Sie eine Einleitung, in der Sie erklären, worum es in dem Text geht.

7. Verwenden Sie präzise und beschreibende Über- und Unterschriften.

8. Verwenden Sie Aufzählungen, um Übersicht zu schaffen.

9. Schreiben Sie verständliche und gut strukturierte Sätze.

10. Verwenden Sie Wörter, die der Leser verstehen kann. (vgl. Språkrådet, 2014)

2 Hiermit ist gemeint, dass der Autor des Textes herausfinden soll, für welche Institution er den Text in KS schreibt, damit er dies entsprechend kommunizieren kann. 


\section{Zielgruppen und Anwendungsbeispiele}

\section{Leichte Sprache}

Die primäre Zielgruppe für LS sind Menschen mit Lernschwierigkeiten und geistigen Behinderungen. Dies geht aus den europäischen Richtlinien für leichte Lesbarkeit hervor. Als rechtliche Grundlage dient ebenfalls die „Verordnung zur Schaffung barrierefreier Informationstechnik nach dem Behindertengleichstellungsgesetz (Barrierefreie-Informationstechnik-Verordnung-BITV2.0)“, welche Internetseiten einer öffentlichen Gewalt dazu verpflichtet, ihr Angebot in Deutsch, Gebärdensprache und Leichter Sprache anzubieten (vgl. Bundesamt für Justiz, 2016).

Das Netzwerk Leichte Sprache nennt allerdings nicht nur Menschen mit Lernschwierigkeiten, sondern auch Menschen mit Demenz und Menschen, die nicht richtig lesen können, nicht sehr gut Deutsch sprechen oder noch dabei sind, die Sprache zu lernen als Zielgruppen (vgl. Netzwerk Leichte Sprache, 2013).

Umgesetzt wird LS zum einen von Webseiten der Bundesregierung, da sie rechtlich dazu verpflichtet sind. Zum anderen gibt es aber auch Vereine, Organisationen und Unternehmen, die ihre Webseiten zusätzlich in LS anbieten. Auch Forschungseinrichtungen wie das KIT bieten Teile ihres Internetauftrittes in LS an (https://www.kit.ed u/leichte_sprache.php).

Die meisten Texte in LS erklären Menschen mit „Lernschwierigkeiten“ ihre Rechte oder beschreiben, wie man am besten in LS schreibt. Darüber hinaus ist es auch wichtig, auf die Informationsbedürfnisse dieser Menschen einzugehen, um sie nicht von alltäglichen Informationen in Tagesnachrichten, Konsumenteninformationen, Freizeitinformationen und (öffentlichen) Verkehrsmitteln auszuschließen (vgl. Freyhoff, 1998).

Tagesnachrichten werden vom Radiosender Deutschlandfunk auf der Webseite nachrichtenleicht.de ${ }^{3}$ angeboten [15.10.2019]. Die Informationen dort sind zwar in Einfacher Sprache verfasst, dennoch richtet sich der Sender explizit an alle - mit Lernschwierigkeiten und ohne (vgl. Deutschlandfunk, o.A.). Es werden aktuelle Geschehnisse in kurzen Artikeln verständlich aufbereitet. Zudem erklärt der Sender das Konzept der Europäischen Union und die Artikel 1-19 des Grundgesetzes in ES.

3 https://www.nachrichtenleicht.de/.

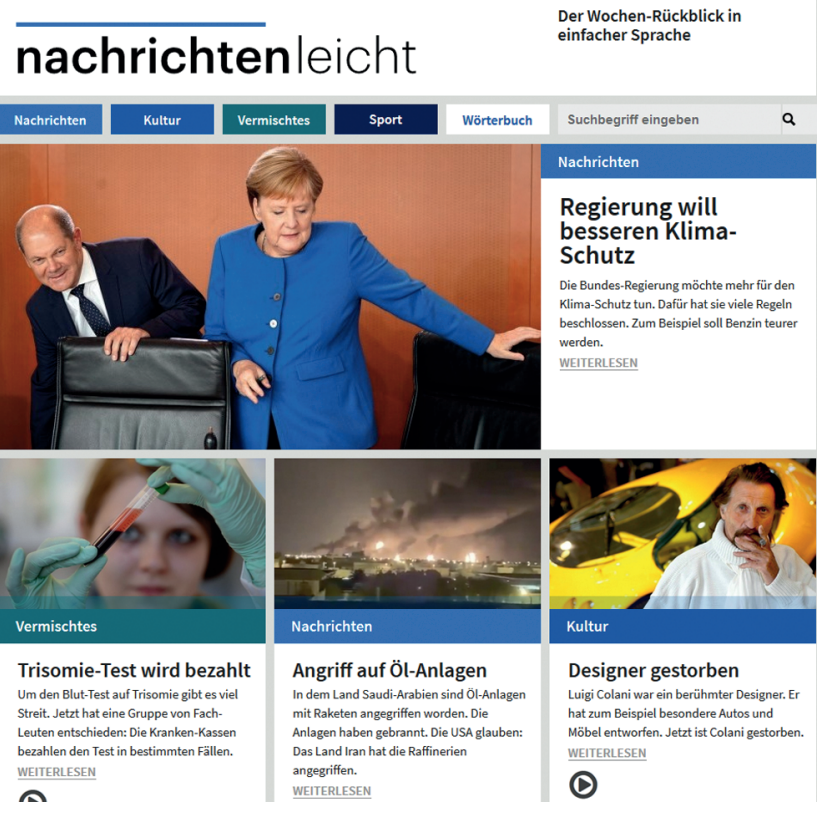

Abbildung 1: Screenshot von https://www.nachrichtenleicht.de/.

Dagegen werden Beipackzettel von Medikamenten, Bedienungsanleitungen oder Formulare nur selten bis gar nicht in LS angeboten. Die Webseite des Deutschen Institut für medizinische Dokumentation und Information (https:// www.dimdi.de/dynamic/de/leichte-sprache/) bietet allerdings die Möglichkeiten in LS Arzt-Diagnosen herauszufinden, nach Festbeträgen für Medikamente zu recherchieren und einen Ratgeber zum Thema „Medikamente im Internet kaufen “. Überweisungsformulare werden von der Sparkasse ${ }^{4}$ in LS erklärt.

Wer in seiner Freizeit gerne im Internet unterwegs ist und dort auf fremde Wörter stößt, der kann diese im Hurraki Wiki ${ }^{5}$ für LS nachschlagen. Bis vor einigen Jahren gab es eine Webseite (www.fahrschule-ohne-barrieren.de), die es ermöglichte, die einzelnen Fragen der theoretischen Führerscheinprüfung in schwerer und leichter Sprache zu lesen. Eine Liste mit weiteren Webseiten in LS findet man unter https://www.bmas.de/DE/Leichte-Sprache/Seiten-1 eichte-Sprache/seiten-leichte-sprache.html.

\section{Klart Språk}

Dagegen richtet sich das KS-Konzept an die Allgemeinheit. Ziel ist eben nicht, einen besonderen Teil der Gesell-

\footnotetext{
4 https://www.sparkasse.de/service/barrierefrei/leichte-sprache/ue berweisung-dauerauftrag-lastschrift.html [15.10.2019]. 5 https://www.hurraki.de/wiki/Hauptseite [15.10.2019].
} 
schaft, sondern möglichst alle anzusprechen. Dabei entsteht allerdings eine sehr heterogene Leserschaft, in der es große Unterschiede im Wissen, den Lesegewohnheiten, der Bildung und den sozialen Schichten gibt. Daher kann ein Text in KS auf viele verschiedene Arten geschrieben sein (vgl. Falck-Ytter, 2009). Menschen mit mentaler Beeinträchtigung oder Lernschwierigkeiten spielen hierbei keine besondere Rolle. Sie sind eine Zielgruppe von vielen (vgl. UiO University of Oslo, 2018). Die Texte unterscheiden sich also nicht wegen der verschiedenen Zielgruppen, sondern aufgrund der unterschiedlichen Motivationen, die hinter dem Text stehen.

Generell dient KS zur Verbesserung der Kommunikation im öffentlichen Raum. Daher ist nicht nur die Regierung dazu angehalten, ihre Texte zusätzlich in $\mathrm{KS} \mathrm{zu}$ schreiben, sondern auch Firmen. Ein bekanntes Anwendungsbeispiel für KS ist die Kommunikation zwischen Schulen und den Eltern der (zukünftigen) Schüler der Skedsmo Kommune (vgl. Språkrådet, 2016).

Für den alltäglichen Informationsbedarf lassen sich keine zu LS vergleichbaren Beispiele wie nachrichtenleicht.de oder hurraki.de finden. Dies könnte zum einen daran liegen, dass es keine eindeutige Zielgruppe gibt, auf die ein Bereich der Informationen angepasst werden muss. Zum anderen kann es aber auch dem geschuldet sein, dass Texte in KS optisch nicht so leicht erkennbar sind, vor allem für Nicht-Muttersprachler, wie Texte in LS.

\section{Meinung der Gesellschaft}

\section{Leichte Sprache}

„Dass ich die Briefe vom Amt lesen kann, ohne dass mir erst jemand erklären muss, was da drin stehen tut. Wenn die Sätze so lang sind, dann ist das für mich zu schwer. Dann habe ich schon gar keine Lust mehr, weiter zu lesen. Aber wenn die Sätze kurz sind, und die Schrift ist schön groß, dann kann ich das auch gut lesen."

- Josef Ströbl, 58, Experte in eigener Sache, Prüfer für Leichte Sprache (Bundesministerium für Arbeit und Soziales, 2018)

„Ich kann keine Zeitung lesen. Und ich brauche meine Betreuerinnen. Sie müssen mir vieles vorlesen. Leichte Sprache ist wichtig! Dann kann ich beim Fußball mitreden. Zum Beispiel weil ich die Regeln verstehe. Und ich kann die SpeiseKarte lesen. Dann kann ich alleine Essen gehen.“
- Nicole Papendorf, Mitarbeiterin im Büro für Leichte Sprache der Lebenshilfe Bremen (Bundesministerium für Arbeit und Soziales, 2018)

Wie aus den beiden Meinungen hervorgeht, kann LS durchaus im Alltag behilflich sein, bei Briefen vom Amt oder in der Freizeit. Es ermöglicht ein Stück weit Selbstbestimmung und Unabhängigkeit von anderen.

Dennoch gibt es Kritik an LS. Diese formulierten beispielsweise Teilnehmende der LeiSA Studie (Leichte Sprache im Arbeitsleben), einem interdisziplinären Forschungsprojekt der Universität Leipzig, das LS auf ihre Tauglichkeit geprüft hat. Die Ergebnisse der Studie sollen als Praxisempfehlungen für den Umgang mit LS dienen (vgl. Bock, 2018). Bemängelt wurde besonders die Regel, zusammengesetzte Wörter mit Bindestrich zu schreiben, da sie nicht vertraut erscheinen und so schlechter wiedererkannt wurden (vgl. Bock, 2018). Die Teilnehmenden sagen u.a.:

„Kennt man nicht [...] künstlich [...] ungewohnt.“ (Bock, 2018)

„Wer ne Schulbildung genossen hat, der weiß ja, dass es zusammengehört.“ (Bock, 2018)

Ebenfalls wurde die Gestaltung von Texten in LS kritisiert. So konnten Teilnehmende der Studie nicht eindeutig erkennen, um welche Textart es sich handelte, wenn ihnen ein Zeitungsartikel in LS gezeigt wurde. Jedoch waren sie durchaus in der Lage, einen normalen Zeitungsartikel als solchen zu identifizieren. Dies zeigt, dass es einen Bedarf gibt, LS Texte so zu gestalten, dass Leser diese auch identifizieren können (vgl. Bock, 2018).

Doch nicht nur die Regeln und die Gestaltung von LS Texten bergen Kontroversen, auch die Adressierung ist innerhalb der Gesellschaft umstritten. Es stellen sich die Fragen, wie „geistige Behinderung“ definiert wird und wer letztendlich entscheidet, wann ein Mensch geistig behindert ist. Deshalb wird in LS-Kreisen häufig der Ausdruck „Menschen mit Lernschwierigkeiten“ verwendet. Doch auch hier stellen sich die gleichen Fragen (vgl. Bock, 2018).

Als der Deutschlandfunk seine Webseite nachrichtenleicht.de 2013 ankündigte, brach eine hitzige Diskussion über ES und LS aus. Einige reagierten mit „Deutschlandfunk für Deppen?“ (Bundeszentrale für politische Bildung, 2014), andere wiederum unterstützten die Idee, auch Menschen mit geringeren Sprachfähigkeiten am politischen und kulturellen Leben teilhaben lassen zu wollen. Wieder andere richteten sich mit ihrer Abneigung weniger gegen die Zielgruppe selbst als gegen den Informationsverlust, der mit dem Umwandeln in ES und LS einhergehe (vgl. Bundeszentrale für politische Bildung, 2014). 
Ein weiterer Kritikpunkt an LS ist, dass sie an Kindersprache erinnert. Auch dies stimmt nur bedingt, da es einige Unterschiede zwischen Sprache für Kinder und LS gibt. Bei Kindersprache werden beispielsweise buntere Seiten mit unterschiedlichen Schriftgrößen verwendet. Meist haben die Seiten einen pädagogischen Hintergedanken, um Kindern Dinge und Wertvorstellungen beizubringen. Zudem sind die Themenwahl und Darstellung ausschlaggebend für die Komplexität eines Textes. Bei LS sind die Sätze kurzgehalten, für Kinder gibt es Haupt- und Nebensätze. In LS werden Bindestriche verwendet, um zusammengesetzte Wörter $\mathrm{zu}$ trennen, bei Kindern nicht. (vgl. Bundeszentrale für politische Bildung, 2014).

Innerhalb der linguistischen Praxis und Forschung gibt es ebenfalls geteilte Meinungen über LS. Zum einen wird die Umsetzung als mangelhaft gesehen, da es noch an empirischen Überprüfungen fehlt und der Ansatz des Konzeptes noch nicht fundiert genug ist. Zum anderen werden die eigentlichen Vermittlungs- und Brückenaufgaben kritisiert, die nur teilweise umgesetzt werden (vgl. Bock, 2015).

Ein weiterer wichtiger Kritikpunkt bezieht sich auf die Auswahl der Themen. Da LS primär für Menschen mit Lernschwierigkeiten gedacht ist, beziehen sich die Texte hauptsächlich auf die Beeinträchtigung selbst und damit verbundene Themen. In der Theorie ist LS für alle, in der Praxis jedoch meistens nicht (vgl. Bock, 2018).

\section{Klart Språk}

Eine Teil-Zielgruppe von KS, die zur Diskussion steht, sind Menschen mit Norwegisch als Fremdsprache. Allerdings gibt es keine Studien, die belegen, dass KS für diese Menschen ein geeignetes Kommunikationsmedium ist (vgl. Holund, 2016). Man geht davon aus, dass ein Text in einer einfachen Sprache für Menschen, die Norwegisch lernen, besser verständlich ist und sie die Sprache schneller lernen. Dennoch gibt es Argumente, die gegen die Vereinfachung von Texten für Menschen in der Lernphase sprechen (vgl. Holund, 2016). So heißt es beispielsweise, durch die Vereinfachung könnte der Lernfortschritt hinausgezögert werden. Ein weiteres Argument dagegen wäre, dass die Texte nicht der mehrheitlichen Realität entsprechen und den Wortschatz, welchen die Menschen in KS lernen, einschränkt (vgl. Holund, 2016).

Wenn man allerdings bedenkt, dass für 20 Prozent der norwegischen Bevölkerung selbst KS noch zu kompliziert ist, würde sich empfehlen, diese Menschen als separate Zielgruppe zu sehen, auf die besser eingegangen werden sollte (vgl. UiO University of Oslo, 2018). In einem Artikel der Zeitschrift Sakprosa ${ }^{6}$ heißt es sogar, dass KS für diejenigen, die damit arbeiten müssen, zu einem Problem geworden ist, da auf dem Gebiet noch nicht ausreichend geforscht wurde (vgl. Sakprosa, 2015). Diese Kritik scheint sowohl für LS (vgl. Bock, 2015) als auch KS (vgl. Sakprosa, 2015) zu gelten.

Abgesehen von den Kritikpunkten von Holund, UiO und Sakprosa findet KS dennoch große Zustimmung, vor allem bei der Regierung und deren Vertreter (vgl. FalckYtter, 2009). So sagt die Ministerin für Erneuerung und Verwaltung, Heidi Grande Røys: „Statstilsette skal skrive så folk forstår. Eit demokrati kan ikkje fungere viss ikkje folk forstår informasjon frå det offentlege.“ (Falck-Ytter, 2009). Dies bedeutet, dass Staatsbeamte so schreiben sollten, dass die Menschen sie verstehen. Denn eine Demokratie funktioniert nur, wenn die Bürger öffentliche Informationen auch begreifen.

\section{Fazit und Ausblick}

Die Unterschiede der beiden Konzepte sind von vornherein klar definiert: LS dient der Barrierefreiheit, KS der Demokratieförderung. Auch die Zielgruppen unterscheiden sich wesentlich. Während LS eine spezielle Zielgruppe ansprechen soll, ist KS für die ganze Bevölkerung. Dennoch findet man zu beiden Konzepten ähnliche Kritik, nämlich, dass sie einen Verlust an Sprach- und Informationsqualität bedeuten. Doch eine wichtige Gemeinsamkeit zeigt sich doch, beide, LS und KS, sind darauf ausgerichtet, den Zugang zu Informationen zu erleichtern.

Der Vergleich der Konzepte dient der Vorbereitung auf den letzten der vier Workshops des Gemeinschaftsprojektes „Aspects of Linguistic Complexity“ der Hochschule Darmstadt und der Universität Trondheim, der nun im Herbst 2019 vor Ort in Norwegen stattfinden wird. Dabei sollen die Unterschiede und Gemeinsamkeiten der Konzepte LS und KS präsentiert und diskutiert werden. Zudem sollen Texte, wie beispielsweise Wikipedia-Einträge, über einen Browser-PlugIn mit Wortlisten hinterlegt werden, um ein einfacheres Lesen zu ermöglichen. Dies geschieht sowohl für Texte in Deutsch als auch für Texte in Norwegisch.

Ziel des Projektes ist es, ein regelbasiertes System zur automatischen Übersetzung von Texten in LS und KS aufzubauen. Für die norwegische Variante soll eine Applika-

6 Die Zeitschrift Sakprosa ist ein akademisches E-Journal für nordische Sakprosa-Forschung. Sakprosa hat mehrere Bedeutungen, hier sind damit ,nicht-fiktive' Texte wie Artikel, Berichte, etc. gemeint (vgl. Sakprosa, 2015). 
tion implementiert werden, die dies ermöglicht. (vgl. Hochschule Darmstadt, 2018)

Ein nächster Schritt könnte sein, die öffentliche Meinung über KS vor Ort durch qualitative Interviews zu erfassen, um so ein Stimmungsbild zu generieren. Dies könnte hilfreich sein, um festzustellen, ob auch außerhalb der Literatur das Bedürfnis nach einer speziellen Zielgruppe, wie der von LS, besteht.

\section{Literatur}

Baumert, A. (2016): Leichte Sprache - Einfache Sprache. Literaturrecherche Interpretation Entwicklung. Hannover. S. 67.

Bock, B. (2015). Barrierefreie Kommunikation als Voraussetzung und Mittel für die Partizipation benachteiligter Gruppen - Ein (polito-)linguistischer Blick auf Probleme und Potenziale von „Leichter“ und „einfacher Sprache“. Linguistik Online, 73(4). S. 121.

Bock, B.M. (2018): „Leichte Sprache“ - Kein Regelwerk. Sprachwissenschaftliche Ergebnisse und Praxisempfehlungen aus dem LeiSA-Projekt. Leipzig. S. 3; 9-11; 28; 40-41; 70.

Bundesministerium für Arbeit und Soziales (2018): Leichte Sprache. Ein Ratgeber. https://www.bmas.de/SharedDocs/Downloads/ DE/PDF-Publikationen/a752-ratgeber-leichte-sprache.pdf? blob=publicationFile. [22.8.2019]

Bundesamt für Justiz (2016): Verordnung zur Schaffung barrierefreier Informationstechnik nach dem Behindertengleichstellungsgesetz. http://www.gesetze-im-internet.de/bitv_2_0/BJNR1843 00011.html. [22.8.2019]

Bundeszentrale für politische Bildung; Kellermann, G. (2014): Leichte und Einfache Sprache - Versuch einer Definition. https://www. bpb.de/apuz/179341/leichte-und-einfache-sprache-versucheiner-definition. [22.8.2019]

Clarity (o.A.): An international association promoting plain legal language - Clarity in your country. http://clarity-international. net/iplwg.html. [11.9.2019]

Deutschlandfunk (o.A.): Was ist nachrichtenleicht? https://www. nachrichtenleicht.de/was-ist-nachrichtenleicht.2053.de.html [11.9.2019]

Falck-Ytter, K. (2009): Klarspråk - hva er det? En kvalitativ studie av klarspråk og klarspråksarbeid i Norge og Sverige. Dragvoll. NTNU-Trykk. S. 11; 30.

Freyhoff, G.; et. al. (1998): Sag es einfach! Europäische Richtlinien für die Erstellung von leicht lesbaren Informationen für Menschen mit geistiger Behinderung für Autoren, Herausgeber, Informationsdienste, Übersetzer und andere interessierte Personen. http://www.webforall.info/wp-content/uploads/2012/12/EU Richtlinie_sag_es_einfach.pdf. [22.8.2019] S. $12 \mathrm{ff}$.

Grotlüschen, A.; Riekmann, W. (2011): leo. - Level-One Studie. Literalität von Erwachsenen auf den unteren Kompetenzniveaus. Presseheft. Universität Hamburg, Hamburg. S. 4 f.

Hochschule Darmstadt (2018): Aspects of Linguistic Complexitiy. A comparative German - Norwegian Valence Approach to the Creation of Resources for Easy-To-Understand Language. https://projects.fzai.h-da.de/linguistic-complexity/projekt/. [22.8.2019]
Holund, V. (2016): Klarspråk i et andrespråkperspektiv. En teoretisk og empirisk studie av andrespråkleseres møte med skriftlig informasjon fra det offentlige. Unv. Diss. Universitetet $\mathrm{i}$ Oslo.S. 31.

Netzwerk Leichte Sprache (o.A.): Das ist Leichte Sprache. https:// www.leichte-sprache.org/das-ist-leichte-sprache/. [22.8. 2019]

Netzwerk Leichte Sprache (2013): Die Regeln für Leichte Sprache. http://www.leichte-sprache.de/dokumente/upload/21dba_ regeln_fuer_leichte_sprache.pdf. [22.8.2019]

Sakprosa (2015): Klarspråk och klarspråksarbete - ett tema i tiden. https://www.journals.uio.no/index.php/sakprosa/article/ view/1284/1292. [22.8.2019]

Språkrådet (2013a): Hva er klarspråk? https://www.sprakradet.no/ Klarsprak/om-klarsprak/hva-er-klarsprak/. [22.08.2019]

Språkrådet (2016): Informasjon om innmelding i skolen og skoleoppstart. https://www.sprakradet.no/Klarsprak/kommunesek toren/kommuner/skedsmo/velkommen-til-skedsmoskolen/. [11.9.2019]

Språkrådet (2013b): Kvifor klarspråk? https://www.sprakradet.no/ Klarsprak/om-klarsprak/hva-er-klarsprak/Kvifor-klarsprak/. [22.8.2019]

Språkrådet (2014): Utdanningsdirektoratets språkprofil. https:// www.sprakradet.no/Klarsprak/prosjekthjelp/laer-av-andre/ Utdanningsdirektoratet/. [22.8.2019]

UiO University of Oslo (2018): Klart språk og medborgerskap. Abstractsamling. https://www.jus.uio.no/forskning/prosjek ter/klarsprak/arrangementer/konferanser/klarsprak-medbor gerskap/abstractsamling.pdf. [22.8.2019] S. 11.

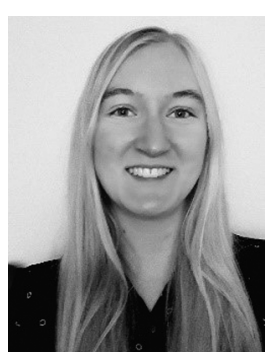

\section{Joane Amrhein}

Hochschule Darmstadt

University of Applied Sciences

Max-Planck-Straße 2

64807 Dieburg

joane.amrhein@stud.h-da.de

Joane Amrhein ist Studentin und wissenschaftliche Mitarbeiterin der Hochschule Darmstadt, University of Applied Sciences. Sie schrieb ihre informationswissenschaftliche Hausarbeit im Rahmen ihres Bachelorstudiums Informationswissenschaft zum Thema Leichte Sprache und Klart Språk. 